\title{
A REVIEW ON MOBILE DATA COLLECTOR IN WIRELESS SENSOR NETWORKS
}

\author{
Mohan BA ${ }^{1}$ and Dr. Sarojadevi $\mathrm{H}^{2}$
}

\begin{abstract}
Wireless Sensor Network (WSN) is progressing as state of art solution for wide range of applications of monitoring, event detection and target tracking in domains such as precision agriculture, pollution prevention, buildings health monitoring, intrusions, fire/flood emergencies and surveillance. The conventional sensor network architecture comprises of densely deployed static nodes over a network area, leading to the problem of network isolation as the connecting node dies in the network. To address this problem WSN architectures are exploiting mobility. This paper discusses WSNs with Mobile Elements for data collection and identifies the corresponding issues and challenges. While mobility involves additional complexity, with the present day deployment of WSN mobility is essential.

Keywords - WSN, mobile data collector, mobile node discovery, motion control, routing.
\end{abstract}

\section{INTRODUCTION}

Wireless Sensor Networks (WSNs) are collection of sensor nodes, which are limited in battery capacity, communication range and computation. Sensor nodes primarily perform three basic operations: (i) sense the environment[1] (ii) process sensed data and (iii) transfer processed data wirelessly to the sink node. The conventional WSNs are composed of static nodes for collecting data and transmitting it to the sink. Introducing mobile node for data collection in static sensor networks is advantageous for several reasons as mentioned below.

- $\quad$ Connectivity - Dense network is changed to sparse networks in which mobile nodes take care of connectivity.

- Reliability - Conventional WSNs are dense and the sensor nodes use multi-hop for transmitting data to sink, which is reduced by use of mobile nodes. So reliability is compromised by collisions, which leads to loss of overall network energy and data.

- $\quad$ Energy Efficiency - Traffic generated by sensor nodes are converge casting, i.e., nodes nearer to sink are used for relaying data from other nodes to sink, due to which this intermediate nodes lose its energy very soon and leads to premature energy depletion causing funneling effect in the network, this problem can be overcome by the use of mobile nodes.

Mobile nodes[2][3][4] in WSN also introduce substantial challenges, which are not present in conventional WSN.

- $\quad$ Contact Detection - ME detection precedes data communication.

\footnotetext{
${ }^{1}$ Department of Computer Science and Engineering Nitte Meenakshi Institute of Technology, Bangalore, Karnataka, India 2 Department of Computer Science and Engineering Nitte Meenakshi Institute of Technology, Bangalore, Karnataka, India
} 
- $\quad$ Mobility aware power management - mobile elements detection can be further optimized by knowing the mobility pattern.

- $\quad$ Mobility Control - A policy has to be defined for motion of mobile elements that can be controlled.

This paper surveys on Mobile Elements (MEs) in WSNs and specifically focuses on data collection and issues that are related to mobility like problem of sensing coverage and connectivity.

\section{WSN ARCHITECTURE WITH MOBILE NODES}

WSN-ME architecture shown in figure 1 contains set of sensor nodes and a sink. The job of sensor nodes is to monitor the physical environment[1] and forward sensed data to base station for further processing. Introducing the mobile node in the architecture acts as intermediate data collector to relay the information from the sensor node to a sink improving the connectivity between the nodes and prolonging the network lifetime.

WSN-ME is a network, where it posses one or more mobile nodes. Normal sensing node or sink can be a mobile node or vice versa. These types of architectures are called three-tier network architecture, where sensor nodes send the data to mobile node from mobile node to sink; the details are analyzed in the following section.

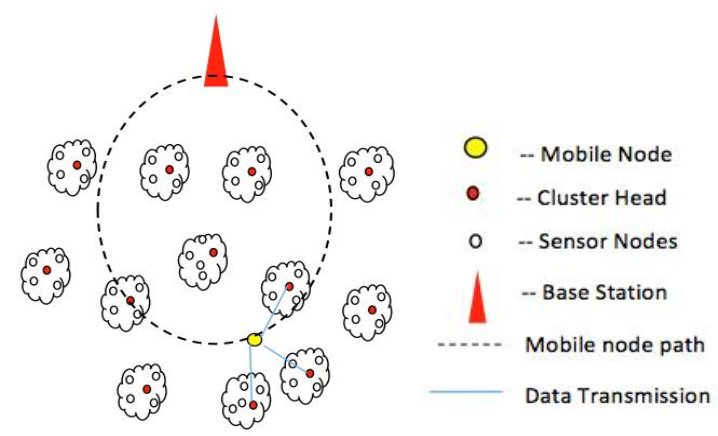

Figure 1: WSN-ME Architecture

\subsection{Re-locatable mobile nodes}

One of the techniques to incorporate Mobile nodes is allowing them to change their location[5][6] in the network to replace the failed node for continuing the service. They only change their location in the network for better connectivity or coverage purposes. WSN architecture with mobile nodes is depicted in Figure 2. This system with re-locatable nodes is used for topology management.

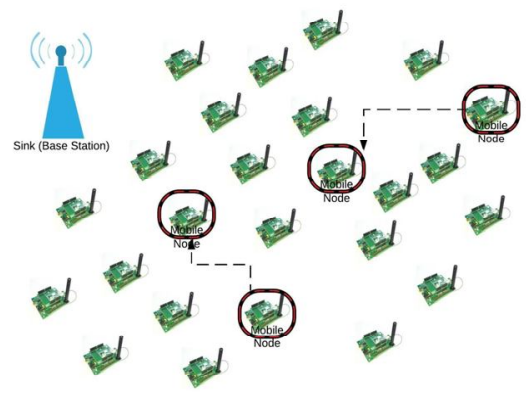

Figure 2: Re-locatable node in the WSN network 


\subsection{Mobile Data Collectors (MDCs)}

In this approach mobile nodes, termed as MDC are deployed for data collection in the network and forward it to base station. Two categories of MDC schemes, which are mobile sink and mobile relay, are discussed as follows.

Mobile Sinks (MSs): Sink nodes are the endpoints of data communication in WSN. The mobile $\operatorname{sink}[7][8]$ in WSN architecture is depicted in Figure 3.

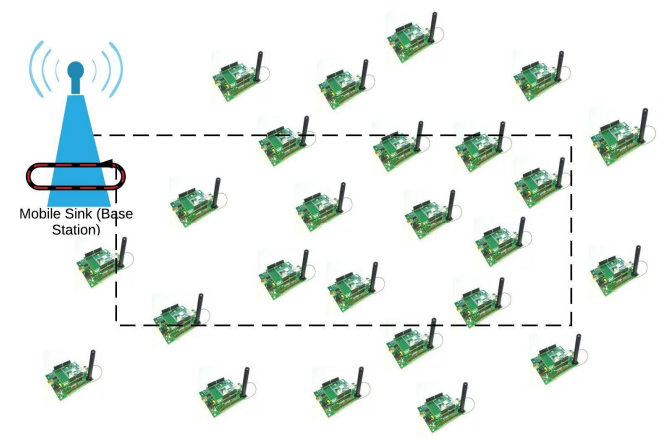

Figure 3: Mobile Sink moving in the WSN network

Mobile Relays (MRs): Mobile Relays are used as support elements for gathering information from sensor nodes and carry it to sinks, acting as intermediate relays as shown in figure 4 . The Mobile Relays based WSN is shown in Figure 4. Mobile Relays assisted data gathering is also called data MULE for WSN[9].

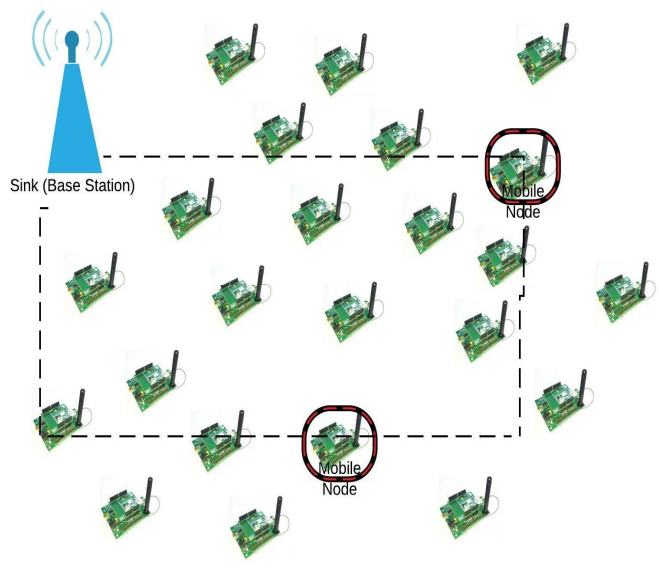

Figure 4: Mobile Relays in the WSN network

2.3 Mobile peers: Another variant to the above approach is mobile peers. In this mobile nodes take the role of data originator as well as relays of the messages in the network. When a peer[10] is in contact with sink, it transfers data gathered from other peers also. Below figure 5 shows mobile peers in WSN. 


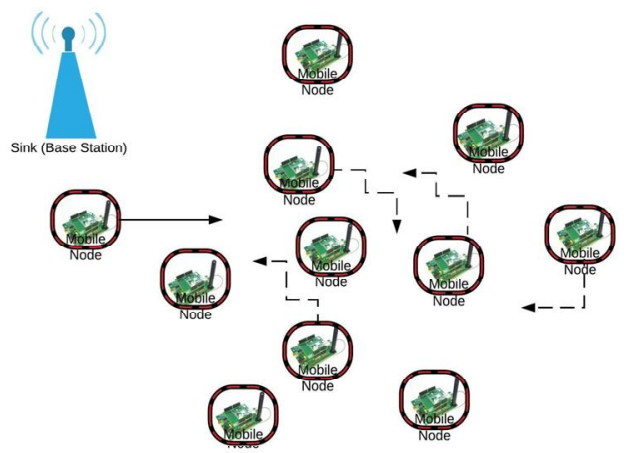

Figure 5: Mobile Peers in the WSN network

\section{DATA COLLECTION IN WSN-MES}

The different stages of data gathering process[11][8][12][13] and issues involved in it are discussed below.

There are three steps in data collection namely mobile node discovery, data transfer and routing.

a. Discovery - Presence of MEs is checked in the contact area by discovery protocols using low energy technique.

b. Data transfer - Efficient transfer protocols are used for getting the maximum information out of contact time.

c. Routing - Routing is a processor of finding optimal multi-hop paths to the destination.

\subsection{Influence of mobility on WSN}

Node mobility has an impact on data collection and depending upon ME mobility control, two main patterns can be defined namely deterministic and random mobility pattern.

Deterministic mobility pattern is illustrated by regularity in the contact of mobile node with sensor node at a specific time.

Random mobility pattern is described by contact taken place in a distribution probability.

\section{DISCOVERY OF MOBILE NODE}

The process of discovering existence of the ME in its contact area is called discovery. Data exchange is possible only during contact period. So to exploit the contact time, discovery should take place at right time and at right place. Two complementary approaches are suggested for discovery with reduced energy consumption.

a. Mobility-Independent discovery protocols, which detect presence of MEs, irrespective of their mobility patterns.

b. Knowledge based power management protocols; sensor nodes will be active only at the time when the ME is expected to be in contact area.

\subsection{Mobility Independent discovery protocols}

These protocols is categorized into three schemes like scheduled rendezvous, on-demand and asynchronous. 
Scheduled rendezvous scheme assumes mobile element and sensor nodes to agree upon specific time to meet for data collection. This is possible, only if MEs follow a strict schedule. In this scheme, mobile elements are boarded in public transportation vehicle for visiting sensor nodes according to predefined schedule. An alternate approach is to synchronize through Global Positioning System (GPS) to wake up accordingly and can contact the neighbors. This approach is implemented in ZebraNet[14] which is simple to implement and efficient also because they only exchange data on schedule basis.

On-demand schemes have nodes, which use multiple radios to discover the mobile node in its range. Long-range radios are used for data communication, while low-range radios are used for awakening seeping nodes. The static sensor nodes continuously monitor the channel for activity with low power radios, conserving the energy. When an activity is detected in the channel, it activates high-power radios and starts communicating with mobile element.

Asynchronous schemes define patterns for sleep/wakeup activity for communication. This scheme discusses variants of asynchronous schemes in which mobile element sends periodic discovery messages, while the static nodes regularly wakes up and listens for advertisements. If it detects discovery message from mobile node then it starts transferring the data to mobile node, else goes to sleep mode.

\subsection{Knowledge-based power management}

The power of sensor nodes can be managed efficiently if the knowledge of mobility pattern of mobile node is exploited properly. If sensors know when the ME is likely to be in contact, it can perform the discovery and rest of the time it can go to sleep.

\section{DATA TRANSFER}

Following the discovery, data transfer takes place with the help of data collection[15] protocol. These protocols are made aware of the issues related to mobility, communication processes and distance from source to destination, which changes with respect to time based on their speed. Results in literature show that robotized mobile element moving slowly will collect considerable amount of data, which is actually independent of the speed. Loss rate minimizes as the ME is approaching the sensor nodes.

\section{ROUTING DATA TO MOBILE ELEMENTS}

This section highlights different approaches for routing data toward MEs[12]. If a path for mobile node is not controllable, then the routing protocol has to be adaptive, otherwise it can not cope up to the mobility. There are two types of routing techniques for uncontrollable mobile nodes namely flat routing and proxy-based routing.

\subsection{Flat Routing}

Many routing protocols for WSN-MEs has been extended from WSNs, one of them is Directed Diffusion (DD)[16], which is prominent for data dissemination in static WSN. DD comprises three stages, in first phase; the sink initiates a query message for an event. In the second phase, which disseminates query in the network and gradients are set up for receiving and forwarding data, which results in multiple paths. The final phase consists of reinforcement, in which path for data propagation is chosen. MintRoute[16] is another approach for flat routing, which can be extended to WSN-MEs called MobiRoute. In this, tree like structure is built for data gathering and paths are evaluated based on metric to minimize the number of retransmissions. 


\subsection{Proxy based routing}

In this technique, special sensor nodes called anchors or rendezvous points gather data and send to nodes called as proxy nodes. When the ME is in its contact, the proxy node starts transmitting data to it. This technique is adopted by Two-Tier Data Dissemination (TTDD) architecture[17], which builds overlay-forwarding structure based on grid for routing data to mobile elements. The intersection of grid lines are called rendezvous/meeting points, while squares are referred to as cells. Upon sending a message, source node determines crossing point and it then starts sending announcement to adjacent intersections.

The meeting points are exploited for minimizing data propagation in a network. The nodes closer to destination intersection will process the message. These recursive announcements will elect the dissemination nodes, which operate as a proxy and establish routing path. The mobile element start data gathering process by flooding a query message to its local cells. The dissemination nodes propagate the query along the grid to source and get data back.

A reinforcement learning based solution is proposed as Hybrid Learning-Enforced Time Domain Routing (HLETDR)[18]. The node nearer to mobile element is designated as proxy, who keeps track of arrival time of mobile node and derive a reinforcement value. These values are calculated as probability of the mobile element being in the vicinity, which is then propagated by the mobile elements to the rest of network nodes through multi-hop paths.

\section{MOTION CONTROL}

The mobility of a node can be either controllable or uncontrollable[19][20]. If node mobility is controllable, then the ME mobility[2] pattern is designed to achieve optimal performance. If node mobility is uncontrollable, sensor nodes can only confirm to the fixed path. Data collection scheme is mainly influenced by mobility pattern. Different data collection strategies are discussed below.

\subsection{Trajectory Control}

Trajectory control[19][21] is classified as static trajectory and dynamic trajectory control. The former refers to path that does not change with respect to time. On the other hand, dynamic trajectory refers to change of mobile element trajectory on the fly, in order to satisfy specific constraints on data collection scheme.

\subsubsection{Static Trajectory.}

Literature has given feasible solutions to address the design of static trajectory for mobile elements. Single mobile node gathers data in WSN, which uses shortest path routing for mobility to conserve the energy. Nodes, which are in inner circle, will use shortest path routing, while other nodes follow a hybrid-forwarding scheme. Integer linear programming is used for determining the path for MEs to visit limited number of feasible sites.

\subsubsection{Dynamic Trajectory}

Dynamic trajectory is divided into priority-based and on-demand categories. The Priority-based scheme depends on constraints on buffer overflow at sensor nodes or latency occurred due to data collection. Another method is Mobile Element Scheduling (MES)[22] where nodes work with different sampling rates. The challenge faced by MES is to schedule the movement of mobile element to prevent buffer overflow at the source node.

On-demand schemes change the trajectory of the mobile node, as soon as static node detect an event. Node Initiated Message Ferrying (NIMF)[23] uses this approach, where ME moving along the route periodically broadcasts its location. A node in its contact area requests ME to be 
visited. ME immediately after receiving the request, modify its trajectory and moves towards the requesting node and then comes back to default route.

\subsection{Speed Control}

The mobile node visits the cluster head following a fixed path to collect the buffered data. The time spent at cluster head for collecting data is called contact time and the place is called contact area. Contact time is decided by the amount of data buffered at that cluster head and this contact time changes with respect to message generation rate and amount of data. This process will affect the latency of the data collection process. To deal with speed of mobile node different algorithms such as Stop to Collect Data (SCD), Adaptive Speed Control (ASC) are explored.

\subsection{Hybrid schemes}

In this scheme, the path and speed of the mobile elements are combined. A range of solutions is derived based on movement and sojourn. The mobile element start from specific location and moves through network, stopping for a given amount of time at specified location for collecting data. Hence, both trajectory and visiting time of the mobile element are predefined.

\section{CONCLUSIONS}

On considering the raise of mobile nodes usage in WSN, recent studies have shown sufficient support for mobile nodes usage in WSN. Mobile nodes in network can conserve the energy of the nodes, however some issues related to mobile node discovery and data transfer have not been clearly addressed and have scope for future research.

This paper characterized the mobile data collection scheme in WSNs. The core architecture for WSN-ME is presented. Furthermore, Data collection schemes are discussed in depth along with challenges. Our investigation explores the scope of research in this area. Mobility in WSN is a revolution compared to static WSN. In this review routing with MEs is found as a common strategy in all WSN-ME schemes. Very few implementation scenarios exist. Real time deployments and evaluation need in-depth investigation. Comprehensive solutions are yet to be proposed, which can be applied to specific application scenarios.

\section{REFERENCES}

[1] T. H. F. Khan, "Mobile Collector aided Energy Reduced ( MCER ) Data Collection in Agricultural Wireless Sensor Networks," 2016 IEEE 6th Int. Conf. Adv. Comput., pp. 629-633, 2016.

[2] O. In, "Mobility-Based Communication in," IEEE Commun. Mag., no. July, pp. 56-62, 2006.

[3] P. Queen, Mobile data collectors in wireless sensor networks Waleed Al-Salih. 2009.

[4] G. Anastasi, M. Conti, and M. Di Francesco, "Data collection in sensor networks with data mules: An integrated simulation analysis," in 2008 IEEE Symposium on Computers and Communications, 2008, pp. 1096-1102.

[5] F. Yin, Z. Li, and H. Wang, "Energy-efficient data collection in multiple mobile gateways WSN-MCN convergence system," 2013 IEEE 10th Consum. Commun. Netw. Conf. CCNC 2013, pp. 271-276, 2013.

[6] B. M. SAROJADEVI H, "A Hybrid Approach for Data collection using Multiple Mobile Nodes in WSN (HADMMN) B,” IEEE Int. Conf. Recent Trends Electron. Inf. Commun. Technol., pp. 736-739, 2016.

[7] N. Gupta and V. Gupta, "A review on sink mobility aware fast and efficient data gathering in wireless sensor networks," in 2016 International Conference on Advances in Computing, Communication, \& Automation (ICACCA) (Spring), 2016, pp. 1-4.

[8] M. R. R, "A Survey on Mobile Data Gathering in Wireless Sensor Networks - Bounded Relay," Int. J. Eng. Trends Technol., vol. 7, no. 5, pp. 205-208, 2014.

[9] S. K. Singh, P. Kumar, and J. P. Singh, "An Energy Efficient Odd-Even Round Number Based Data Collection using Mules in WSNs,” pp. 1255-1259, 2016.

[10] E. Rondini and S. Hailes, "Load Sharing and Bandwidth Control in Mobile P2P Wireless Sensor Networks."

[11] S. Hanoun, D. Creighton, and S. Nahavandi, "Decentralized mobility models for data collection in wireless sensor networks," in 2008 IEEE International Conference on Robotics and Automation, 2008, pp. 10301035.

[12] A. Sayyed, G. M. de Araujo, and L. B. Becker, "Smart data collection in large scale sparse WSNs," in 2016 
9th IFIP Wireless and Mobile Networking Conference (WMNC), 2016, pp. 1-8.

[13] C. Wang and H. Ma, "Data collection with multiple controlled mobile nodes in wireless sensor networks," Proc. Int. Conf. Parallel Distrib. Syst. - ICPADS, pp. 489-496, 2011.

[14] B. Zhang and F. Yu, "An event-triggered localization algorithm for mobile wireless sensor networks," 2010 2nd Int. Conf. Futur. Comput. Commun., pp. V1-250-V1-253, 2010.

[15] N. Brinis, M.-A. Koulali, L. A. Saidane, P. Minet, and A. Kobbane, "Strategic data gathering in wireless sensor networks," in 2016 International Wireless Communications and Mobile Computing Conference (IWCMC), 2016, pp. 547-552.

[16] M. A. Rassam, A. Zainal, and M. Al-shaboti, "A Sinkhole Attack Detection Scheme in Mintroute Wireless Sensor Networks," 1st IEEE Int. Symp. Telecommun. Technol., pp. 71-75, 2012.

[17] H. Luo, F. Ye, J. Cheng, S. Lu, and L. Zhang, "TTDD $\square$ : A Two-tier Data Dissemination Model for Largescale Wireless Sensor Networks $\square$, , Springer, vol. 11, no. 1, pp. 161-175, 2005.

[18] P. Baruah, R. Urgaonkar, and B. Krishnamachari, "Learning-enforced time domain routing to mobile sinks in wireless sensor fields," Proc. - Conf. Local Comput. Networks, LCN, pp. 525-532, 2004.

[19] A. A. Somasundara et al., "Controllably Mobile Infrastructure for Low Energy Embedded Networks," IEEE Trans. Mob. Comput., vol. 5, no. 8, pp. 958-973, 2006.

[20] S. Basagni, A. Carosi, and C. Petrioli, "Controlled Vs. uncontrolled mobility in wireless sensor networks: Some performance insights," IEEE Veh. Technol. Conf., pp. 269-273, 2007.

[21] L. Xue, D. Kim, Y. Zhu, D. Li, W. Wang, and A. O. Tokuta, "Multiple heterogeneous data ferry trajectory planning in wireless sensor networks," in IEEE INFOCOM 2014 - IEEE Conference on Computer Communications, 2014, pp. 2274-2282.

[22] A. A. Somasundara, A. Ramamoorthy, and M. B. Srivastava, "Mobile element scheduling with dynamic deadlines," IEEE Trans. Mob. Comput., vol. 6, no. 4, pp. 395-410, 2007.

[23] K. K. Ahmed, M. H. Omar, and S. Hassan, "Survey and Comparison of Operating Concept for Routing Protocols in DTN," J. Comput. Sci., 2016. 